\title{
Mit der Modellkette RCP-GCM-RCM-mGROWA projizierte Grundwasserneubildung als Datenbasis für zukünftiges Grundwassermanagement in Nordrhein-Westfalen
}

\author{
Frank Herrmann ${ }^{1}$ (D) Klaus Keuler $^{2}$ (D) Tim Wolters $^{1}$ (D) Sabine Bergmann ${ }^{3} \cdot$ Michael Eisele $^{3}$. \\ Frank Wendland ${ }^{1}$
}

Eingegangen: 11. Mai 2020 / Überarbeitet: 15. Oktober 2020 / Angenommen: 17. Dezember 2020 / Online publiziert: 5. Januar 2021

(c) Der/die Autor(en) 2021

\section{Zusammenfassung}

Mit einem Multi-Modell-Ensemble wurde analysiert, wie sich der Klimawandel auf den Grundwasserhaushalt in Nordrhein-Westfalen (NRW) auswirkt. Hierzu wurden Projektionen der zukünftigen Grundwasserneubildung für insgesamt 36 Mitglieder der Modellkette RCP-GCM-RCM-mGROWA, bestehend aus 3 RCP-Szenarien zukünftiger globaler Erwärmung, 6 globalen und 5 dynamischen regionalen Klimamodellen sowie dem Wasserhaushaltsmodell mGROWA, vorgenommen. Mit dem Ensemble wurden für die hydrogeologischen Großräume NRWs nur teilweise signifikante Änderungen der jährlichen Grundwasserneubildung in den Perioden 2011-2040, 2041-2070 und 2071-2100 projiziert. Ein Robustheitstest mit zwei Kriterien (Übereinstimmung und Signifikanz der Änderungssignale) liefert keine belastbare Begründung dafür, dass sich die Grundwasserneubildung bis 2100 systematisch und signifikant ändern wird. Aus statistischer Perspektive wird deshalb die Schlussfolgerung gezogen, dass in NRW langfristig eine Grundwasserneubildung erwartet werden kann, die sich nicht grundlegend vom Niveau der Periode 1971-2000 unterscheidet. Hydro-meteorologisch befindet sich NRW in einer Übergangszone, in der eine Zunahme der Winterniederschläge die Wirkung der Erwärmung auf die Grundwasserneubildung wahrscheinlich kompensiert.

Schlüsselwörter Grundwasserneubildung $\cdot$ Nordrhein-Westfalen $\cdot$ mGROWA $\cdot$ Klimawandel $\cdot$ Multi-Modell-Ensemble Robustheit

Frank Herrmann

f.herrmann@fz-juelich.de

Klaus Keuler

klaus.keuler@b-tu.de

Tim Wolters

t.wolters@fz-juelich.de

Sabine Bergmann

sabine.bergmann@lanuv.nrw.de

Michael Eisele

michael.eisele@lanuv.nrw.de

Frank Wendland

f.wendland@fz-juelich.de

1 Institut für Bio- und Geowissenschaften -

IBG 3 Agrosphäre, Forschungszentrum Jülich, Wilhelm-Johnen-Straße, 52428 Jülich, Deutschland

2 Fakultät 2, Fachgebiet Atmosphärische Prozesse, Brandenburgische Technische Universität (BTU) Cottbus-Senftenberg, Burger Chaussee 2, 03044 Cottbus, Deutschland

3 Landesamt für Natur, Umwelt und Verbraucherschutz Nordrhein-Westfalen, Leibnizstr. 10, 45659 Recklinghausen, Deutschland 


\title{
Groundwater recharge in North Rhine-Westphalia projected using the model chain RCP-GCM-RCM-mGROWA
}

\begin{abstract}
In order to analyze the impact of climate change on groundwater resources in North Rhine-Westphalia a multi-model ensemble for projecting future groundwater recharge was established. The ensemble consists of 36 members of the model chain RCP-GCM-RCM-mGROWA in total, i.e. combinations of 3 greenhouse gas concentration trajectories, 6 global and 5 regional climate models, and the water balance model mGROWA. The ensemble projections show only a few significant changes of groundwater recharge in the future periods 2011-2040, 2041-2070, and 2071-2100. A robustness test using the two-criteria model agreement and the significance of the individual model projections did not reveal systematic and significant changes of groundwater recharge until 2100. From the statistical point of view, groundwater recharge can be expected to remain at the current level. Hydrometeorological, North Rhine-Westphalia is located in a transition zone in which the impact of the rising winter precipitation on groundwater recharge is counter-balanced by the impact of warming.
\end{abstract}

Keywords Groundwater recharge $\cdot$ North Rhine-Westphalia $\cdot$ mGROWA $\cdot$ Climate change impact $\cdot$ Multi-model ensemble $\cdot$ Robustness

\section{Einführung}

In den vergangen 10 Jahren wurde in nahezu allen Regionen Nordrhein-Westfalens (NRW) ein anhaltender Trend fallender Grundwasserstände beobachtet, welcher im Herbst 2019 in historischen Tiefstständen des Grundwassers mündete. Parallel dazu fiel die Grundwasserneubildung niedriger aus, als im langjährigen Mittel der Periode 1981-2010 (Herrmann et al. 2020). Als Erklärung dafür wird eine Überlagerung natürlicher Klimavariabilität mit den bereits beobachtbaren Auswirkungen des anthropogenen Klimawandels vermutet. Mit dem EURO-CORDEX-Ensemble (Jacob et al. 2014) wurde im Mittel für NRW bis zum Ende des 21. Jahrhunderts gegenüber der Periode 1971-2000 ein Anstieg der Jahresmitteltemperatur im Bereich zwischen 1,5 und $4,3{ }^{\circ} \mathrm{C}$ sowie eine Zunahme der Jahresniederschläge im Bereich zwischen 1 und $25 \%$ projiziert (LANUV o.J.). Die Veränderungen von Temperatur und Niederschlag werden wahrscheinlich saisonal und auch regional unterschiedlich stark ausgeprägt sein. Damit stellt sich die Frage, ob sich der Klimawandel negativ auf den Grundwasserhaushalt und damit auf das Grundwasserdargebot auswirken könnte, was im Extremfall zu Anpassungen in der Grundwasserbewirtschaftung führen müsste. Insbesondere ein langfristiger flächendeckender Rückgang der Grundwasserneubildung würde für einige Regionen des Landes ein verändertes Nutzungsregime notwendig erscheinen lassen, damit dort die Grundwasserressourcen erhalten bleiben.

Die zukünftige Konstanz statistischer Kennzahlen zum Landschaftswasserhaushalt wird schon seit längerer Zeit infrage gestellt (z.B. Milly et al. 2008). Vor diesem Hintergrund würde es auch für die Grundwasserbewirtschaftungsplanung in NRW nicht mehr ausreichen, nur den Status quo fortzuführen, z. B. durch periodische Aktualisierungen bei der Grundwasserneubildungsermittlung. Vielmehr müs- sen belastbare, statistisch abgesicherte Szenarien und Modellketten entwickelt werden, welche die Schaffung einer Datenbasis zur quantitativen Entwicklung des Wasserhaushalts und damit der Grundwasserressourcen zum Ziel haben (Merz et al. 2012). Dazu sollten Modelle eingesetzt werden, welche die Grundwasserneubildung nicht lediglich in ein lineares Verhältnis zum Niederschlag setzen, sondern in starkem Maße in Abhängigkeit von vorhandenem Bodenwasser und zeitlicher Verteilung der Niederschläge berechnen (Himmelsbach und Neukum 2018). Zur Schaffung einer solchen Datenbasis ist die Projektion der Grundwasserneubildung mithilfe eines Multi-Modell-Ensembles bestehend aus globalen Emissionsszenarien, globalen und regionalen Klimamodellen sowie eines hydro(geo)logischen Impaktmodells das gegenwärtige Mittel der Wahl (Holman et al. 2012). Dieser Ansatz ist in der Vergangenheit bereits in einer Vielzahl hydrologischer Studien und bundesdeutschen Regionen verfolgt worden (z.B. Hattermann et al. 2015; Huang et al. 2010; Menzel et al. 2006). Auf Ebene der Bundesländer sollten Schlussfolgerung aus derartigen Studien im Hinblick auf aktuell als verlässlich eingestufte Klimaprojektionen kontinuierlich überprüft werden (Scheihing 2019).

Im Rahmen der hier dokumentierten Studie kam als hydro(geo)logisches Impaktmodell das in NRW auf Landesebenen etablierte Wasserhaushaltsmodell mGROWA (Herrmann et al. 2015) zum Einsatz. Mit mGROWA können die Grundwasserneubildung und weitere Abflusskomponenten in hoher zeitlicher und räumlicher Auflösung (Tagesschritte, $100 \mathrm{~m}$ Raster) berechnet werden. Der Beitrag umfasst eine kompakte Vorstellung der Modellkette RCP-GCM-RCM-mGROWA (Multi-Modell-Ensemble), eine einführende Diskussion des Rest-Bias der projizierten Grundwasserneubildung, eine Darstellung der projizierten Änderung der Grundwasserneubildung, eine kurze 
Diskussion der Robustheit der Ensembleprojektion sowie Interpretationen mit Relevanz für das Grundwassermanagement in NRW. Mit den Ergebnissen der Studie verfügt das Land NRW über eine Datenbasis für zukünftiges Grundwassermanagement, basierend auf aktuell als verlässlich eingestuften Klimaprojektionen.

\section{Methodik der Modellkette RCP-GCM-RCM- MGROWA}

Für die Projektion zukünftig möglich erscheinender Grundwasserneubildungshöhen in NRW wurde ein Multi-ModellEnsemble etabliert. Dieses Ensemble besteht aus insgesamt 36 Mitgliedern der Modellkette RCP-GCM-RCMmGROWA, deren Elemente und Verknüpfungen in den folgenden Unterkapiteln in ihren wichtigsten Grundzügen vorgestellt werden. Die tatsächlich im Rahmen der hier präsentierten Studie durchgeführten Simulationen betreffen die Impaktmodellierung mit dem Wasserhaushaltsmodell mGROWA (Herrmann et al. 2013). Die Ergebnisse aus den mGROWA vorgelagerten Klimamodellen sind im Rahmen einer Vielzahl von Projekten entstanden und in den hier verwendeten Kombinationen frei verfügbar über das Deutsche Klimarechenzentrum (DKRZ o.J.). An der Schnittstelle zwischen den RCMs und mGROWA erfolgt eine BiasKorrektur der simulierten täglichen Niederschlagssummen, die Berechnung einer täglichen Summe der potenziellen Evapotranspiration über Gras aus den simulierten atmosphärischen Zustandsgrößen und der Strahlungsbilanz sowie ein räumliches Downscaling vom RCM-Gitter auf das mGROWA-Modellraster.

\section{Klimaprojektionen}

Die RCP-Szenarien (Moss et al. 2010) geben in der Modellkette die möglich erscheinende globale Erwärmung vor. Hinter RCP (Representative Concentration Pathways) stehen Annahmen für eine zukünftig weiter veränderte Strahlungsbilanz der Erdatmosphäre, die aus steigenden Treibhausgaskonzentrationen resultiert. RCP2.6 impliziert einen Strahlungsantrieb von maximal $3 \mathrm{~W} / \mathrm{m}^{2}$ vor 2100 und nach diesem Maximum einen Rückgang der Treibhausgaskonzentrationen. Im RCP4.5 wird eine Stabilisierung des Strahlungsantriebs bei $4,5 \mathrm{~W} / \mathrm{m}^{2}$ angenommen, d.h. die Treibhausgaskonzentrationen verbleiben in der zweiten Hälfte des 21. Jahrhunderts dauerhaft auf einem erhöhten Niveau. Das RCP8.5 steht für einen weiterhin kontinuierlich ansteigenden Strahlungsantrieb bis auf $8,5 \mathrm{~W} / \mathrm{m}^{2}$ am Ende des Jahrhunderts, der aus relativ ungebremst steigenden Treibhausgaskonzentrationen resultiert.

Die RCP-Szenarien liefern Randbedingungen für Globale Klimamodelle (GCM, General Circulation Model) wel- che wiederum die Randbedingungen für räumlich höher auflösende Regionale Klimamodelle (RCM, Regional Climate Model) bereitstellen. Für den Antrieb des Wasserhaushaltsmodells mGROWA in der Modellkette wurde ein durch die Europäische Initiative EURO-CORDEX (Jacob et al. 2014) geschaffenes und dann im Verbundprojekt ReKliEsDe (Huebener et al. 2017) erweitertes Ensemble aus GCMRCM-Kombinationen verwendet. Dieses Ensemble wurde jedoch aufgrund der vom Deutschen Wetterdienst (DWD) publizierten Empfehlungen reduziert (DWD o.J.). Es umfasst derzeit nur noch dynamische RCMs, deren prinzipielle Funktionsweise in Knoche und Keuler (2017) vorgestellt wird. Die Ensemblemitglieder (RCP-GCM-RCM-Kombinationen) sind in Tab. 1 gelistet.

Die Verkettung von GCMs mit RCMs wird auch als dynamische Regionalisierung bezeichnet (Knoche und Keuler 2017). GCMs bilden die wichtigsten physikalischen Prozesse in der Atmosphäre der Erde ab, die in ihrer Wirkung über längere Zeiträume das Klima bestimmen. GCMs rechnen üblicherweise mit einer Gitterweite von ca. $200 \mathrm{~km}$. Die resultierenden globalen Verteilungen klimatischer Größen sind jedoch zu grob, um daraus lokal und kleinregional verlässliche Schlussfolgerungen zu ziehen. Aus diesem Grund werden in die Gitterstrukturen der GCMs die RCMs verschachtelt (engl. nested), d.h. die GCMs liefern die atmosphärischen Randbedingungen an den Grenzen der RCMs. Diese simulieren ebenfalls die wichtigsten physikalischen Prozesse in der Atmosphäre einer größeren Region (z.B. Europas), derzeit typischerweise auf einer Gitterweite von ca. $12 \mathrm{~km}$. Als Ergebnis resultieren für die Gitterpunkte hochaufgelöste Zeitreihen atmosphärischer Größen (häufig auf Stundenbasis), wie zum Beispiel Niederschlagsmengen, oberflächennähe Lufttemperaturen, die Komponenten des Strahlungshaushaltes, die Komponenten des Windfeldes, etc., die im Falle des EURO-CORDEX-Ensembles die Zeit bis Ende 2100 umfassen. Aus diesen Größen muss für hydrologische Impaktmodelle geeigneter klimatischer Input berechnet werden.

\section{Bias-Korrektur und Downscaling der Klimaprojektionen}

Die mit Klimamodellen für einen Referenzzeitraum simulierten räumlichen und zeitlichen Niederschlagsmuster weisen gegenüber Beobachtungsdaten meistens einen sogenannten Bias auf, d.h. die Modelle über- oder unterschätzen beobachtete langjährige Mittelwerte und in vielen Fällen auch die saisonalen Verteilungsmuster täglicher Niederschlagshöhen. Die durch aktuelle RCMs projizierten Niederschlagsmuster weisen im Vergleich zu den antreibenden GCMs jedoch schon einen systematisch reduzierten Bias auf (z. B. Sørland et al. 2018), der dennoch in hydrologischen und hydrogeologischen Klimafolgestudien einen 
Tab. 1 Zusammensetzung des Ensembles aus RCP-GCM-RCM-Kombinationen. Die Abkürzungen entsprechen der im Projekt ReKliEs-De verwendeten Nomenklatur. Erläuterungen für PBIAS* in \% und RMSE* in mm/a im Text

Table 1 RCP-GCM-RCM combinations of the ensemble. Abbreviations according to the ReKliEs-De-nomenclature. Explanations of PBIAS* in $\%$ und RMSE* in mm/a in the text

\begin{tabular}{|c|c|c|c|c|c|c|}
\hline Szenario & Globalmodell & Lauf & Regionalmodell & Abkürzung & PBIAS* & RMSE* \\
\hline $\mathrm{RCP} 2.6$ & ICHEC-EC-EARTH & 12 & CCLM 4-8-17 & R26-E12-CLM & -10 & 54 \\
\hline $\mathrm{RCP} 2.6$ & MPI-M-MPI-ESM-LR & 1 & CCLM 4-8-17 & R26-MP1-CLM & -12 & 42 \\
\hline $\mathrm{RCP} 2.6$ & ICHEC-EC-EARTH & 12 & KNMI-RACMO22E & R26-E12-RAC & -9 & 49 \\
\hline $\mathrm{RCP} 2.6$ & MOHC-HadGEM2-ES & 1 & KNMI-RACMO22E & R26-HG2-RAC & -7 & 45 \\
\hline $\mathrm{RCP} 2.6$ & ICHEC-EC-EARTH & 12 & SMHI-RCA4 & R26-E12-RCA & \pm 0 & 48 \\
\hline $\mathrm{RCP} 2.6$ & MOHC-HadGEM2-ES & 1 & SMHI-RCA4 & R26-HG2-RCA & +3 & 45 \\
\hline $\mathrm{RCP} 2.6$ & MPI-M-MPI-ESM-LR & 1 & SMHI-RCA4 & R26-MP1-RCA & +5 & 38 \\
\hline $\mathrm{RCP} 2.6$ & MPI-M-MPI-ESM-LR & 1 & REMO2009 & R26-MP1-R09 & -7 & 47 \\
\hline $\mathrm{RCP} 2.6$ & MPI-M-MPI-ESM-LR & 2 & REMO2009 & R26-MP2-R09 & -6 & 52 \\
\hline $\mathrm{RCP} 4.5$ & ICHEC-EC-EARTH & 12 & CCLM 4-8-17 & R45-E12-CLM & -10 & 54 \\
\hline $\mathrm{RCP} 4.5$ & MOHC-HadGEM2-ES & 1 & CCLM 4-8-17 & R45-HG2-CLM & -5 & 47 \\
\hline $\mathrm{RCP} 4.5$ & MPI-M-MPI-ESM-LR & 1 & CCLM 4-8-17 & R45-MP1-CLM & -12 & 42 \\
\hline $\mathrm{RCP} 4.5$ & ICHEC-EC-EARTH & 1 & KNMI-RACMO22E & R45-E01-RAC & -6 & 41 \\
\hline $\mathrm{RCP} 4.5$ & MOHC-HadGEM2-ES & 1 & KNMI-RACMO22E & R45-HG2-RAC & -7 & 45 \\
\hline $\mathrm{RCP} 4.5$ & IPSL-IPSL-CM5A-MR & 1 & SMHI-RCA4 & R45-IP5-RCA & \pm 0 & 40 \\
\hline $\mathrm{RCP} 4.5$ & ICHEC-EC-EARTH & 12 & SMHI-RCA4 & R45-E12-RCA & \pm 0 & 48 \\
\hline $\mathrm{RCP} 4.5$ & MOHC-HadGEM2-ES & 1 & SMHI-RCA4 & R45-HG2-RCA & +3 & 45 \\
\hline $\mathrm{RCP} 4.5$ & MPI-M-MPI-ESM-LR & 1 & SMHI-RCA4 & R45-MP1-RCA & +5 & 38 \\
\hline $\mathrm{RCP} 4.5$ & MPI-M-MPI-ESM-LR & 2 & REMO2009 & R45-MP2-R09 & -6 & 52 \\
\hline RCP8.5 & CCCMa-CanESM2 & 1 & CCLM 4-8-17 & R85-CA2-CLM & -8 & 52 \\
\hline RCP8.5 & ICHEC-EC-EARTH & 12 & CCLM 4-8-17 & R85-E12-CLM & -10 & 54 \\
\hline $\mathrm{RCP} 8.5$ & MOHC-HadGEM2-ES & 1 & CCLM 4-8-17 & R85-HG2-CLM & -5 & 47 \\
\hline RCP8.5 & MIROC-MIROC5 & 1 & CCLM 4-8-17 & R85-MI5-CLM & -10 & 62 \\
\hline $\mathrm{RCP} 8.5$ & MPI-M-MPI-ESM-LR & 1 & CCLM 4-8-17 & R85-MP1-CLM & -12 & 42 \\
\hline $\mathrm{RCP} 8.5$ & ICHEC-EC-EARTH & 1 & KNMI-RACMO22E & R85-E01-RAC & -6 & 41 \\
\hline $\mathrm{RCP} 8.5$ & MOHC-HadGEM2-ES & 1 & KNMI-RACMO22E & R85-HG2-RAC & -7 & 45 \\
\hline $\mathrm{RCP} 8.5$ & IPSL-IPSL-CM5A-MR & 1 & SMHI-RCA4 & R85-IP5-RCA & \pm 0 & 40 \\
\hline RCP8.5 & ICHEC-EC-EARTH & 12 & SMHI-RCA4 & R85-E12-RCA & \pm 0 & 48 \\
\hline RCP8.5 & MOHC-HadGEM2-ES & 1 & SMHI-RCA4 & R85-HG2-RCA & +3 & 45 \\
\hline RCP8.5 & MPI-M-MPI-ESM-LR & 1 & SMHI-RCA4 & R85-MP1-RCA & +5 & 38 \\
\hline $\mathrm{RCP} 8.5$ & MPI-M-MPI-ESM-LR & 1 & REMO2009 & R85-MP1-R09 & -7 & 47 \\
\hline RCP8.5 & MPI-M-MPI-ESM-LR & 2 & REMO2009 & R85-MP2-R09 & -6 & 52 \\
\hline RCP8.5 & CCCMa-CanESM2 & 1 & REMO2015 & R85-CA2-R15 & -6 & 46 \\
\hline $\mathrm{RCP} 8.5$ & ICHEC-EC-EARTH & 12 & REMO2015 & R85-E12-R15 & -5 & 51 \\
\hline RCP8.5 & MOHC-HadGEM2-ES & 1 & REMO2015 & R85-HG2-R15 & -1 & 43 \\
\hline RCP8.5 & MIROC-MIROC5 & 1 & REMO2015 & R85-MI5-R15 & -4 & 49 \\
\hline
\end{tabular}

relevanten Einfluss auf die projizierten Abflusskomponenten haben kann (z.B. Huang et al. 2013). Der räumliche und saisonale Bias des mit dem EURO-CORDEX-Ensemble simulierten Niederschlags ist europaweit für die Periode 1989-2008 von Kotlarski et al. (2014) qualitativ und quantitativ dokumentiert worden. Hübener et al. (2017) stellen den Niederschlags-Bias der RCMs des ReKliEs-DeEnsembles für Deutschland (Periode 1971-2000) detaillierter dar und beschreiben die generelle Problematik der Evaluierung der Qualität der regionalen Klimamodelle. Die saisonale Unter- und Überschätzung einzelner RCMs im deutschlandweiten Mittel liegt demnach in einer bemerkenswerten Bandbreite zwischen -37 und $+50 \%$. Im Hinblick auf die Zielstellung der hier dargestellten Studie wird deshalb von einer Notwendigkeit der Verwendung Bias-korrigierter Niederschläge ausgegangen, ohne den Bias einzelner RCMs für NRW detailliert zu beschreiben. Bezüglich des Bias der aus projizierten Klimasignalen berechneten potenziellen Evapotranspiration über Gras fehlen derzeit noch landesweite detaillierte Untersuchungen. Dieser Bias wird jedoch um eine Größenordnung geringer als der Niederschlags-Bias eingeschätzt. 
Über geeignete Bias-Korrekturverfahren wurde in den vergangenen zwei Dekaden viel diskutiert und ihre Vorbzw. Nachteile in Klimafolgestudien herausgearbeitet. So argumentieren Ehret et al. (2012) beispielsweise, dass durch Bias-Korrekturen die Unsicherheiten in den Modellketten weiter verschleiert werden. Andererseits können in der Modellkette RCP-GCM-RCM-mGROWA, wenn Niederschläge ohne Bias-Korrektur verwendet werden, negative Änderungssignale der Grundwasserneubildung resultieren, die betragsmäßig größer sind, als das Referenzniveau der Grundwasserneubildung in der beobachteten Vergangenheit. In der Interpretation der Änderungssignale würde dies dann ein Absinken der Grundwasserneubildung unter das Niveau Null bedeuten, was schlichtweg unrealistisch wäre.

Vor diesem Hintergrund wurden in der hier präsentierten Modellkette die projizierten Niederschlagssummen Bias-korrigiert verwendet. Der Niederschlags-Bias wurde im Rahmen des Verbundprojektes ReKliEs-De mit dem LOCIVerfahren (Local Intensity Scaling, Schmidli et al. 2006) für die in Deutschland lokalisierten RCM-Gitterpunkte korrigiert. Mit LOCI werden alle Tagesniederschläge einer Si- mulation so angepasst, dass für jeden Kalendertag der klimatologische Witterungsverlauf einer vorgegebenen Referenzperiode (hier 1971-2000) adäquat wiedergeben wird. So reproduzieren die korrigierten Niederschläge an jedem Gitterpunkt des Modellgebietes die klimatologischen Monatsmittelwerte und insbesondere die mittleren saisonalen Muster der Referenzperiode. Die dabei für jeden Kalendertag und jeden Gitterpunkt ermittelten Korrekturfaktoren werden dann auf alle Tagesniederschläge, also auch die der verschiedenen Klimaszenarien, übertragen. Neben der Veränderung der Niederschlagsmenge kann sich dabei auch die Stärke der Niederschlagsänderung zwischen verschiedenen Klimaperioden verändern. Als Referenz wurden hier die HYRAS-Daten (Rauthe et al. 2013) verwendet, für deren Erzeugung Beobachtungsdaten auf die RCM-Gitterpunkte projiziert wurden. Für die Übertragung der Klimadaten (Niederschlag und berechnete potenzielle Evapotranspiration über Gras als Tagessummen) vom RCM-Gitter (ca. $12 \mathrm{~km}$ ) auf das mGROWA-Modellraster $(100 \mathrm{~m})$ wurde im Rahmen dieser Studie die von Marke et al. (2011) vorgeschlagene bilineare Interpolation angewendet (Abb. 1).

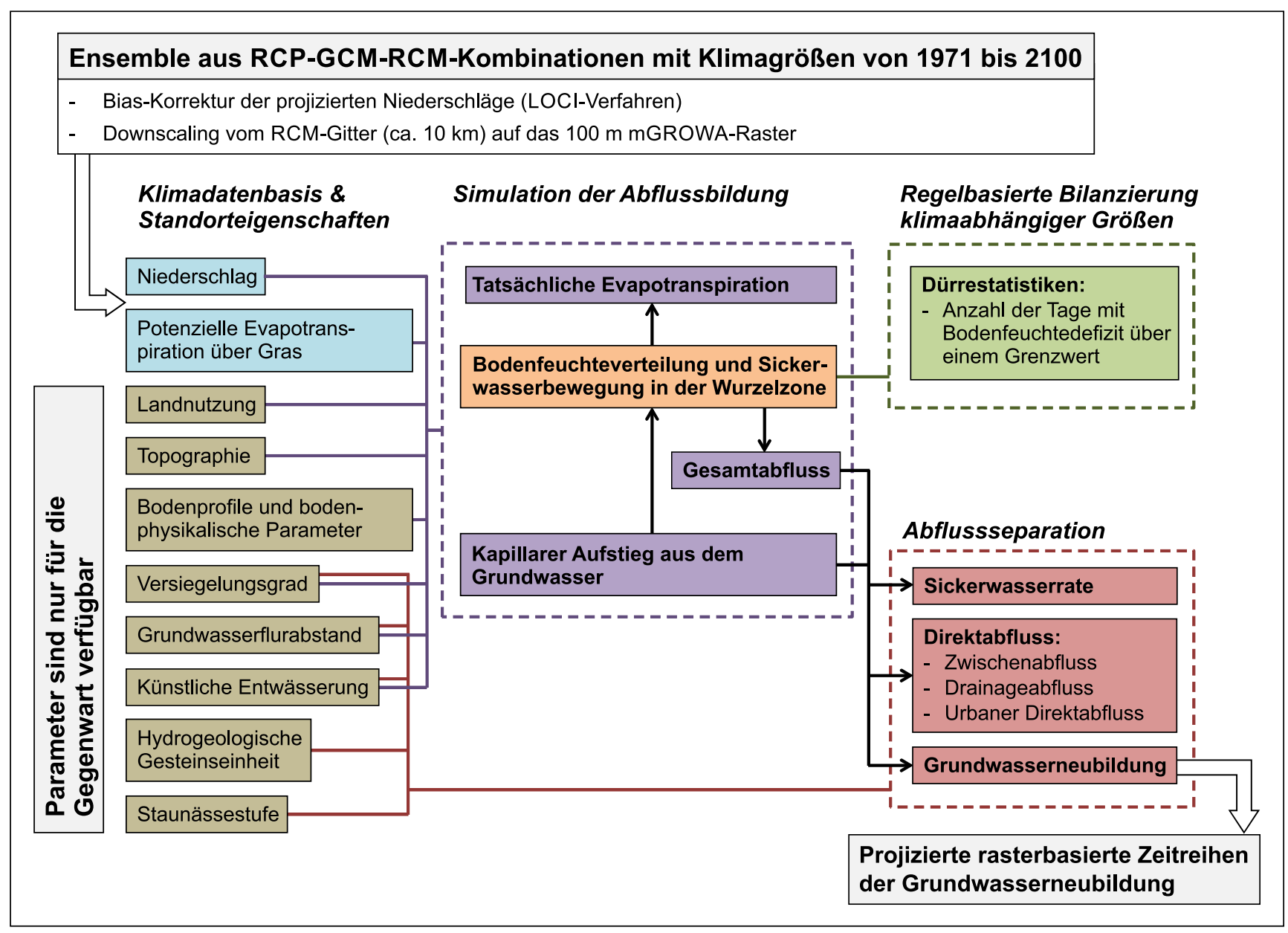

Abb. 1 Konzept des Wasserhaushaltsmodells mGROWA integriert in die Modellkette RCP-GCM-RCM-mGROWA

Fig. 1 Diagram showing the integration of mGROWA in the model chain RCP-GCM-RCM-mGROWA 


\section{Das Wasserhaushaltsmodell mGROWA}

Die räumlich und zeitlich hochaufgelöste Projektion des Wasserhaushaltes erfolgt mit dem letzten Element der Modellkette, dem Wasserhaushaltsmodell mGROWA, in zwei Stufen. Der Simulation der Abflussbildung in Tagesschritten folgt eine Abflussseparation in Monatsschritten, an deren Ende projizierte rasterbasierte Zeitreihen der monatlichen Grundwasserneubildungshöhe stehen. Diese Zeitreihen können für weitere Analysen zu Jahressummen oder langjährigen Mittelwerten aggregiert werden. In der Implementierung für das Bundesland Nordrhein-Westfalen ist das Modell mGROWA detailliert in Herrmann et al. (2015) erläutert. In Herrmann et al. (2013) ist die aus Perspektive der Grundwasserbewirtschaftung relevante Abbildung der Grundwasserneubildung einzelner Monate dargelegt. Im Rahmen der hier vorgestellten Studie wurden alle Parameter (Landnutzung, Jahresgang der Grundwasserflurabstände, etc.) langfristig konstant gehalten, um eine Wirkanalyse der Klimaänderung auf die Situation der Gegenwart zu ermöglichen.

In der prozessorientierten ersten Stufe des Modells wird in Tagesschritten die tatsächliche Evapotranspiration von der Erdoberfläche und die Abflussbildung in Form des Gesamtabflusses flächendifferenziert und instationär berechnet (Abb. 1). Grundlage hierfür ist die allgemeine hydrologische Wasserhaushaltsgleichung. Die Bodenwasserdynamik, repräsentiert durch den Speicherterm der Gleichung, wird mit dem als Modul integrierten Mehrschicht-Bodenwasserhaushaltsmodell BOWAB (Engel et al. 2012) simuliert. Ein derartiges Modul ist essenziell für Projektionen der Grundwasserneubildung mit einem Multi-Modell-Ensemble. Sickerwasserbewegung und damit spätere Grundwasserneubildung kann in relevanter Größenordnung erst stattfinden, wenn im Herbst der Bodenwasserspeicher nahezu auf Feldkapazität aufgefüllt wurde. Das Zusammenspiel von veränderten Niederschlags- und Temperaturmustern im Sommer (stärker ausgeprägte Dürren), Herbst (langsameres Auffüllen des Bodenwasserspeichers) und Winter (wärmer und verspätetes Einsetzen der Grundwasserneubildung) steuert maßgeblich das jährliche Niveau der Grundwasserneubildung und damit die resultierenden Änderungssignale (Herrmann et al. 2017). Das bedeutet, dass höhere Winterniederschläge nicht zwangsläufig in ein höheres Grundwasserneubildungsniveau münden. Dieses Zusammenspiel kann jedoch nur adäquat projiziert werden, wenn alle relevanten Prozesse in einem Bodenwassermodul abgebildet sind.

Mit dem Bodenwassermodul (alle Details in Herrmann et al. 2013) wird im Modell mGROWA die tatsächliche Evapotranspiration aus den durchwurzelten Bodenhorizonten (in NRW als 5 Modellschichten abgebildet) sowie die Sickerwasserbewegung in Abhängigkeit vom Füllstand des
Bodenwasserspeichers bilanziert. Dazu wird eine Vielzahl Parameter verwendet, die aus unterschiedlichen Quellen stammen und für die mGROWA-Modellierung im NRW zusammengestellt wurden. Beispielsweise stammen die den Bodenwasserspeicher charakterisierenden räumlich verteilten Parameter Wassergehalt bei Feldkapazität und Wassergehalt am permanenten Welkepunkt aus Kartenwerken des Landes NRW (BK 1:50.000), viele weitere bodenspezifische Parameter aus Müller und Waldeck (2011) und Renger et al. (2009) sowie vegetationsspezifische Parameter zur Berechnung der tatsächlichen Verdunstung aus ATV-DVWK (2002) und Disse (1995). Der Bilanzraum des Bodenwassermoduls ist durch die durchwurzelte Bodenzone und auf grundwasserbeeinflussten Standorten zusätzlich durch den Grundwasserflurabstand vorgegeben. Aus den Angaben zur Grundnässestufe in der Bodenkarte wurde zur Schaffung dieser unteren Randbedingung ein charakteristischer Jahresgang des Grundwasserflurabstands abgeleitet. Auf dieser Basis sowie der simulierten Bodenwasserspannung (Modell von van Genuchten 1980), werden täglich variierende kapillare Aufstiegsraten aus dem Grundwasser berechnet. Mit dieser Vorgehensweise wird in einer ersten Näherung dieser natürliche Rückkopplungsmechanismus im Modell berücksichtigt.

In der empirischen zweiten Stufe der Simulation des Wasserhaushaltes mit dem Modell mGROWA erfolgt in Monatsschritten die Separation der in der ersten Stufe ermittelten Abflussbildung in die Grundwasserneubildung sowie in mehrere Komponenten des Direktabflusses. Basis für diesen Verfahrensschritt ist die Identifizierung und Parametrisierung der Standorteigenschaften, die für die Auftrennung des gebildeten Abflusses lokal relevant sind. In NRW haben beispielsweise landwirtschaftliche Drainagesysteme und geringe Grundwasserflurabstände in vielen Lockergesteinsgebieten einen erheblichen Einfluss auf die netto stattfindende Grundwasserneubildung. Dieser Situation wird mit entsprechend angepassten Datengrundlagen und Berechnungsschritten Rechnung getragen (Tetzlaff et al. 2008). In den Festgesteinsregionen NRWs wird die Grundwasserneubildung mithilfe kalibrierter BFI-Werte berechnet (Bogena et al. 2005), die flächendifferenziert und abhängig von den hydraulischen Gebirgsdurchlässigkeiten das Verhältnis der Grundwasserneubildung zum Gesamtabfluss wiedergeben. Dabei wird explizit die mit dem Bodenwasserhaushaltsmodul für die Böden über Festgesteinsgrundwasserleitern berechnete monatliche Sickerwassermenge in einen schneller und mit lateraler Strömungskomponente abfließenden Interflow sowie eine tatsächlich den nutzbaren Grundwasserleiter auffüllende Grundwasserneubildung aufgespaltet. Als Interflow wird dabei im Sinne von Bloomfield et al. (2009) und Haberlandt et al. (2001) die schnelle unterirdische Direktabflusskomponente verstanden, die nicht zum Grundwasserdargebot beiträgt. 


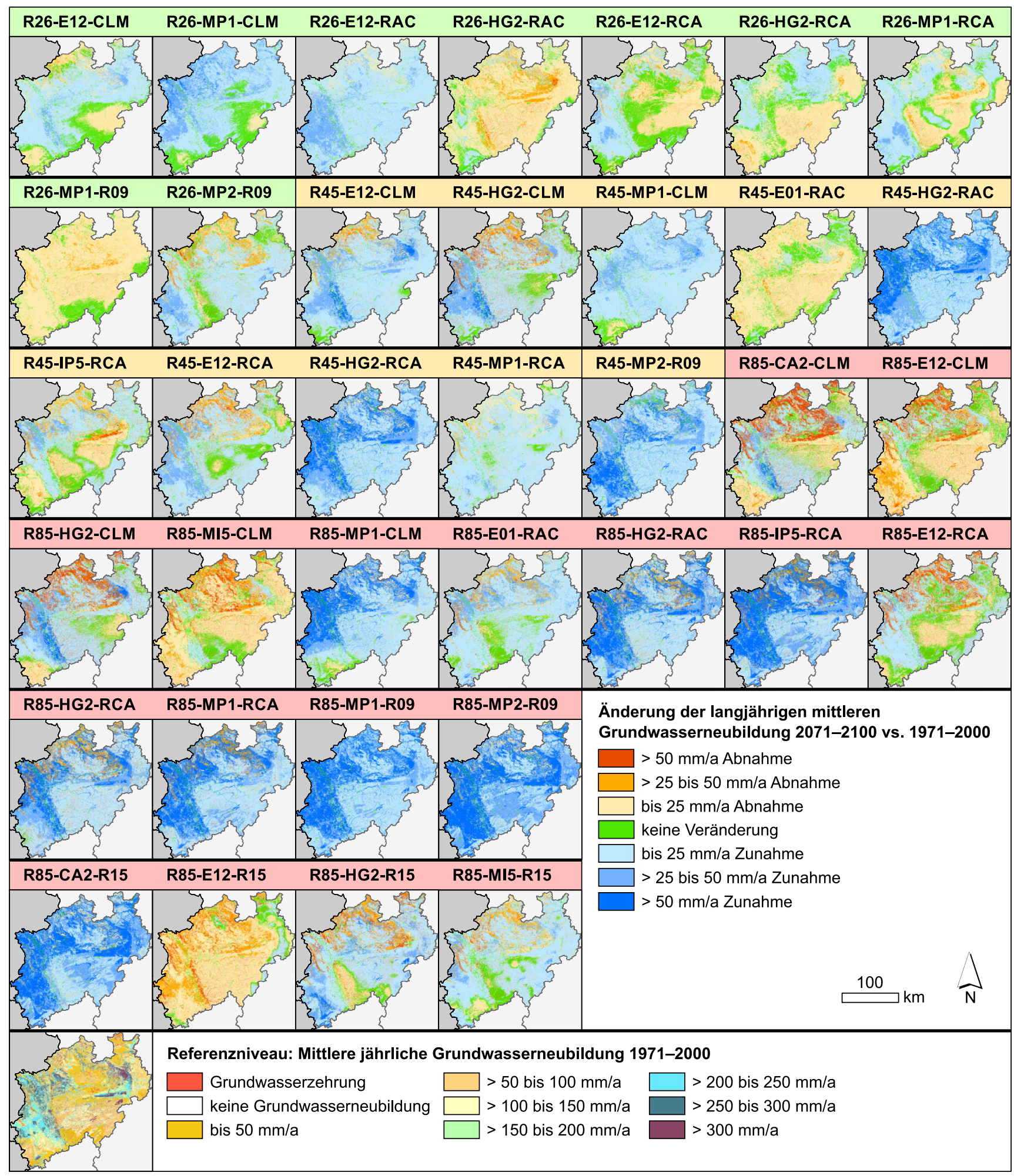

Abb. 2 Räumlich verteilte Änderungssignale der projizierten Grundwasserneubildung (2071-2100 vs. 1971-2000). Unterste Zeile: Das Referenzniveau der Grundwasserneubildung in NRW simuliert auf Basis beobachteter Klimadaten

Fig. 2 Spatial distribution of changing signals of projected groundwater recharge (2071-2100 vs. 1971-2000). Bottom row: reference level of groundwater recharge in NRW simulated based on observed climate data 
Eine Darstellung der räumlichen Verteilung der BFI-Werte in NRW ist in Herrmann et al. (2015) gegeben.

Die mGROWA-Simulationen auf Basis von historischen beobachteten Klimadaten (im Folgenden auch ,,beobachtetes Referenzniveau“) werden standardmäßig anhand langjähriger beobachteter Abflusszeitreihen evaluiert. Die dabei für das Bundeslandes NRW angewendete Methodik ist ausführlich in Herrmann et al. (2015) dargestellt und hinsichtlich der Unsicherheiten diskutiert worden. Im Rahmen der Evaluierung des Modells in 30 Einzugsgebieten wurde für den Gesamtabfluss eine sehr gute Modellperformance erzielt und mit einem Wert von 0,96 für die Nash-Sutcliffe-Modelleffizienz (NSE, flächengewichtet) sowie einer nur sehr geringen Tendenz zur Unterschätzung von $0,9 \%$ (PBIAS, flächengewichtet) nachgewiesen. Für die ebenfalls evaluierte Grundwasserneubildung ist die Modellperformance etwas geringer (NSE 0,55; PBIAS -3,4\%). Auf Basis dieser Kennzahlen wurde die Schlussfolgerung gezogen, dass der Landschaftswasserhaushalt in NRW mit mGROWA insgesamt realistisch abgebildet wird.

\section{Projizierte Änderung der Grundwasserneubildung}

Mit der Modellkette RCP-GCM-RCM-mGROWA wurden instationäre Simulationen der Grundwasserneubildung für den Zeitraum von 1971 bis 2100 durchgeführt. Das resultierende Ensemble umfasst 36 einzelne Projektionen, wobei 9 auf Basis des RCP2.6, 10 auf Basis des RCP4.5 und 17 auf Basis des RCP8.5 durchgeführt wurden (Tab. 1). Von 1971 bis 2005 basieren die Klimaprojektionen auf beobachteten Treibhausgasemissionen. Die 30-Jahresperiode 1971-2000 wird demzufolge als ,projizierte historische Referenzperiode" bezeichnet. Ab 2006 basieren die Projektionen auf den RCP-Szenarien. Die 30-Jahresperiode am Ende des Jahrhunderts von 2071 bis 2100 wird auch als ,,projizierte ferne Zukunft" bezeichnet. Insgesamt werden im Folgenden die Unterschiede zwischen den projizierten historischen Referenzperioden und dem beobachteten Referenzniveau, sowie die Änderungssignale für drei zukünftige 30-Jahresperioden (2011-2040, 2041-2070, 2071-2100) gegenüber der projizierten historischen Referenzperiode vorgestellt.

\section{Projizierte historische Referenzperioden vs. beobachtetes Referenzniveau}

Die Abb. 2 zeigt im unteren Teil das auf Basis beobachteter Klimadaten mit mGROWA für die Periode 1971-2000 bestimmte beobachtete Referenzniveau der langjährigen mittleren Grundwasserneubildung, dessen Evaluierung (im Text oben) erläutert wurde. Gegenüber diesem Niveau weist die mit dem Ensemble für die historische Referenzperiode pro- jizierte Grundwasserneubildung in den einzelnen Projektionen variierend einen auch räumlich unterschiedlich ausgeprägten sogenannten Rest-Bias auf. Dieser Rest-Bias ist durch mehrere sich in ihrer Wirkung überlagernde Ursachen begründet und ein starkes Argument dafür, dass Änderungssignale der Grundwasserneubildung immer innerhalb einer konsistenten Simulation berechnet werden sollten und nicht zwischen einem beobachteten Referenzniveau und einer Projektion.

- Die Klimaprojektionen mit RCMs sind auch nach einer Bias-Korrektur generell nicht zeitpunktgenau. Das heißt, sie bilden nicht die Wetterlagen der Vergangenheit in ihrer zeitlichen Reihenfolge exakt ab, sondern zielen auf die Reproduktion langjähriger Klimastatistiken (z.B. Mittelwerte des Niederschlags). Dabei können die interannuellen Variationen und die saisonalen Variationen eines einzelnen Jahres individuell von realen Abläufen abweichen.

- Für die Bias-Korrektur des Niederschlages wurde mit HYRAS ein anderer Datensatz verwendet als für die mGROWA-Simulation zur Bestimmung des beobachteten Referenzniveaus.

- Zusätzlich weisen weitere projizierte Klimagrößen (z.B. Temperatur, Sonnenscheindauer, Windgeschwindigkeit, etc.) mit Relevanz für die Berechnung der potenziellen Evapotranspiration über Gras (klimatischer Input für mGROWA) einen - wenn auch häufig geringen - Bias auf (z.B. Willkofer et al. 2018), der jedoch in vielen Fällen nicht korrigiert werden kann oder sollte (Erläuterungen in Ehret et al. 2012) und der auch im Rahmen dieser Studie nicht korrigiert wurde.

- Letztendlich sind auch die für die Bias-Korrektur verwendeten Verfahren aus methodischer Sicht immer in ihrer Wirkung begrenzt, d. h. sie sind nicht in der Lage, alle Parameter einer Klimastatistik in gleicher Qualität an die beobachteten Parameter exakt anzupassen (Reproduktion der Extremwerte, saisonale Verteilung und Mittelwerte, korrekte Anzahl an Tagen mit Nieselregen, etc.).

Der Rest-Bias der für die historische Referenzperiode projizierten Grundwasserneubildung variiert räumlich auch aufgrund eines komplexen Zusammenspiels aus den Klimasignalen der RCMs und der Parametrisierung einzelner Standorte in mGROWA. Eine detaillierte qualitative und quantitative Bewertung ist aus wissenschaftlicher Perspektive sinnvoll, kann jedoch im Rahmen dieses Beitrags nur in einer ersten Annäherung erfolgen. Weil die Klimaprojektionen nicht zeitpunktgenau sind, kann eine Bewertung auch nur auf Basis statistischer Kennzahlen erfolgen. Die Tab. 1 listet dazu für alle Ensemblemitglieder die für die jeweilige historische Periode berechneten Kennzahlen PBIAS* und RMSE*. PBIAS (Berechnung in Moriasi et al. 2007) gibt die prozentuale Über- oder Unterschätzung (negativ o. po- 
sitiv) der jährlichen Grundwasserneubildung durch die Projektionen gegenüber dem beobachteten Referenzniveau an. RMSE (Berechnung in Moriasi et al. 2007) ist ein weit verbreitet genutztes Fehlermaß (Quadratwurzel aus der mittleren quadratischen Abweichung) und wird hier zur Charakterisierung der Streuung der projizierten jährlichen Grundwasserneubildung um ihren langjährigen Mittelwert verwendet. Beide Kennzahlen wurden rasterbasiert berechnet. PBIAS* (in \%) und RMSE* (in mm/a) sind die Mediane der resultierenden räumlichen Verteilungen.

Hinsichtlich der prozentualen Über- und Unterschätzung des beobachteten Referenzniveaus befinden sich alle Projektionen im Rahmen einer Spannbreite $(-12$ bis $+5 \%)$, die für hydrologische Modelle im Vergleich zu beobachteten Abflusszeitreihen als gute $(< \pm 15 \%)$ bis sehr gute $(< \pm 10 \%)$ Modellperformance bezeichnet wird (Moriasi et al. 2007). Tendenziell kann demnach der Rest-Bias der projizierten Grundwasserneubildung als relativ gering eingestuft werden, wobei auffällig ist, dass ein größerer Teil der Projektionen zu einer leichten Überschätzung neigt. Der RMSE** des beobachteten Referenzniveaus beträgt ca. $46 \mathrm{~mm} / \mathrm{a}$, d. $\mathrm{h}$. die jährliche Grundwasserneubildung variierte im Medianmittel um $46 \mathrm{~mm} / \mathrm{a}$ um die langjährigen Mittelwerte. Die RMSE* vieler Projektionen liegen in einer ähnlichen Größenordnung, wobei größere Werte als eine stärkere und klei- nere Werte als eine weniger starke Ausprägung von Nassund Trockenperioden interpretiert werden.

Aus methodischer Perspektive trägt die Kenntnis des Rest-Bias einer projizierten Wasserhaushaltsgröße zur Reduzierung eines Teils der Unsicherheiten bei, die weiterhin mit solchen Ensembleprojektionen verbunden sind. In der Praxis sollte jedoch, aufgrund der skizzierten Problemlage, bei der Interpretation weniger der Blick auf das absolute Niveau der projizierten Grundwasserneubildungshöhen in einer zukünftigen Periode gerichtet werden. Vielmehr sollten die Änderungssignale einer Projektion in Bezug zum Referenzniveau (Abb. 2 unten) bewertet werden, um ein absolutes zukünftiges Niveau zu schätzen.

\section{Änderungssignale der projizierten Grundwasserneubildung}

Die mit der Modellkette RCP-GCM-RCM-mGROWA projizierten Zeitreihen der Grundwasserneubildung liegen in hoher räumlicher Auflösung - d.h. in NRW für insgesamt ca. 3,41 Mio. je 1 ha große Rasterzellen - vor. Die Abb. 2 zeigt die Änderungssignale der projizierten Grundwasserneubildung der 30-Jahres-Periode 2071-2100 gegenüber der projizierten historischen Referenzperiode 1971-2000. Alle Projektionen zeigen räumlich differenziert unterschiedliche Änderungen der Grundwasserneubildung an.

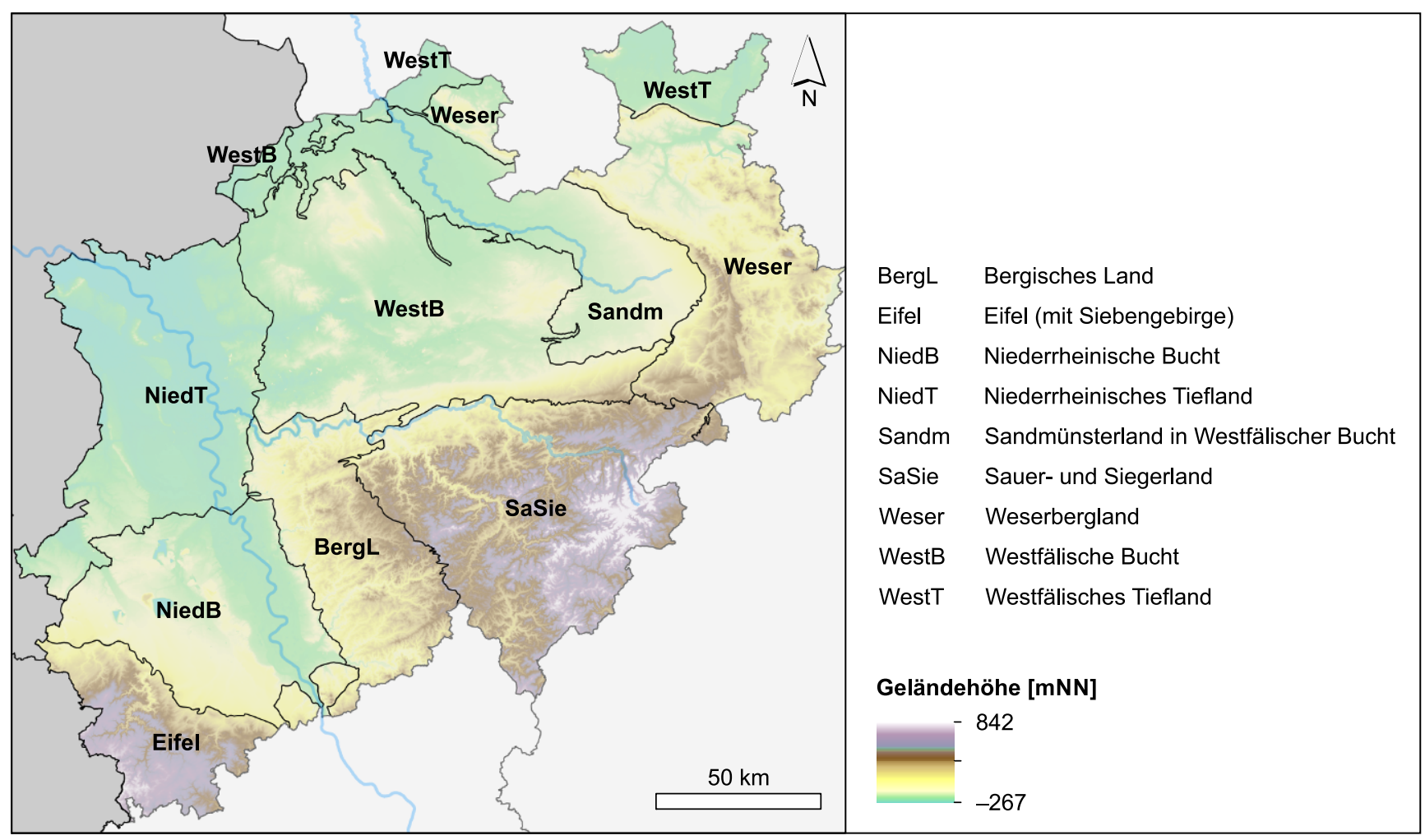

Abb. 3 Hydrogeologische Raumgliederung für eine regionsspezifische Analyse der Ensembleergebnisse

Fig. 3 Hydrogeologically distinct regions in NRW 


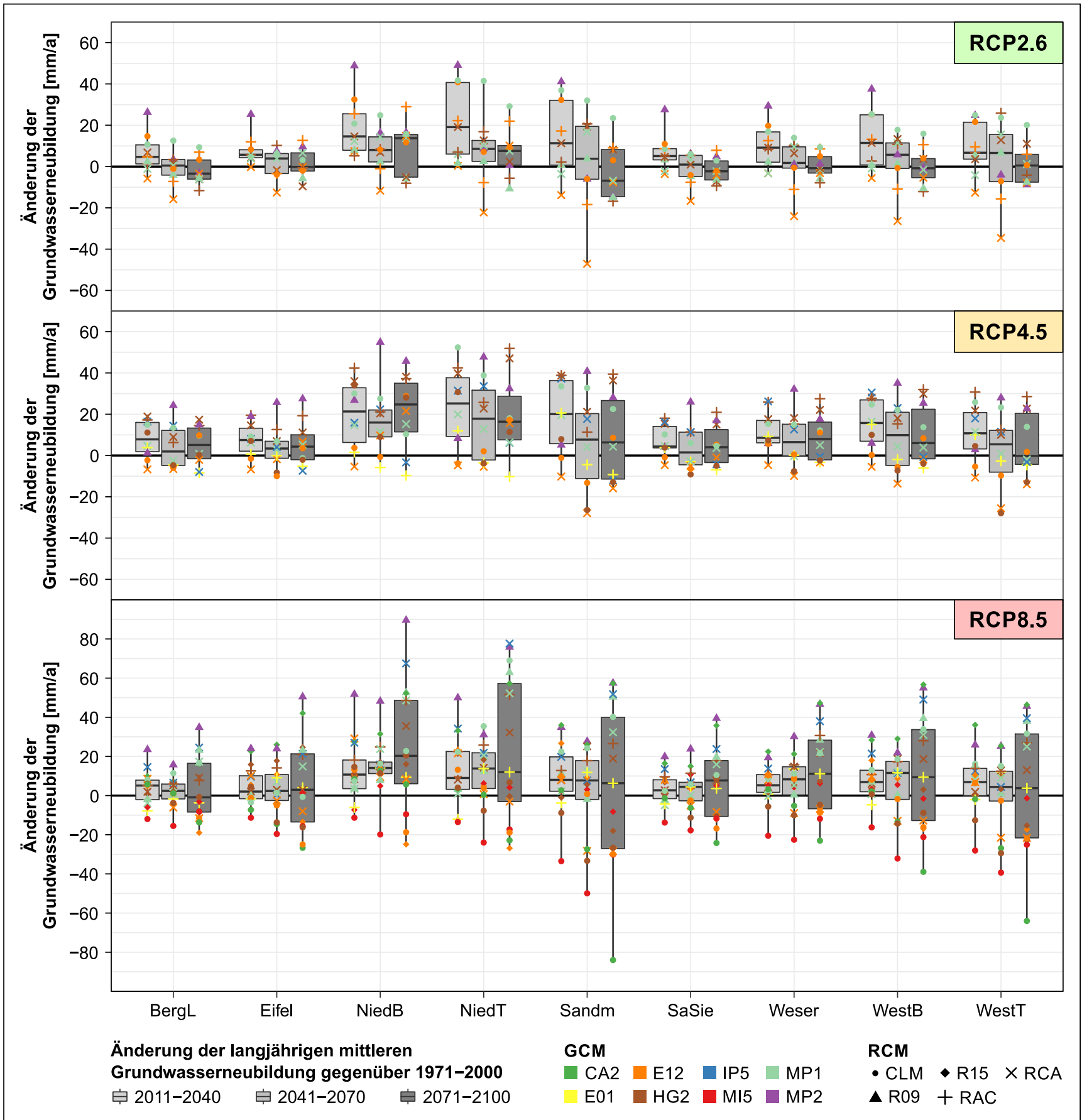

Abb. 4 Boxplot der Änderungssignale der langjährigen mittleren Grundwasserneubildung in den drei RCP-Teilensembles und den drei zukünftigen 30-Jahres-Perioden in den exemplarischen hydrogeologischen Großlandschaften (Benennung wie in Abb. 3). Fette Linien in den Boxen kennzeichnen die Mediane, die Boxen begrenzen den Interquartilsabstand, d. h. 50\% der Werte

Fig. 4 Boxplot of changing signals of long-term mean groundwater recharge in three RCP ensemble parts and three future 30-year periods in the hydrogeologically distinct regions (names in Fig. 3). Bold lines indicate the median, boxes display the interquartile range

Für die Beantwortung von grundwasserwirtschaftlichen Fragestellungen können diese Ergebnisse theoretisch auf beliebige Gebietskulissen aggregiert (gemittelt) werden. Im Rahmen der hier dokumentierten Studie wurde eine solche Aggregierung exemplarisch für die in Abb. 3 dargestellten hydrogeologischen Großlandschaften durchgeführt. Aus hydrogeologischer Perspektive sind die dargestellten Großlandschaften zum Beispiel in Ad-hoc-AG_Hydrogeologie (2016) beschrieben. 
Die auf Ebene der Großlandschaften aggregierten Veränderungen der langjährigen mittleren Grundwasserneubildung in den drei zukünftigen 30-Jahres-Perioden sind, aufgeteilt in RCP-Teilensembles, in Abb. 4 als Boxplots dargestellt. Als Erstes kann festgestellt werden, dass die Mediane in einem engen Bereich um Null streuen, d.h. im Mittel keine gravierende Veränderung der Grundwasserneubildung projiziert wird. Insgesamt zeigen mehr Mediane eine leichte Zunahme gegenüber dem Referenzniveau an, wobei insbesondere im RCP2.6 in der ferneren Zukunft die Mediane auch wieder unter das Referenzniveau fallen. In den Teilensembles RCP4.5 und RCP8.5 sind nahezu alle Mediane positiv. Die Interquartilsabstände variieren einerseits in den Regionen und auch in den verglichenen Perioden. Im RCP2.6 nehmen sie mit der Zeit meist ab, wohingegen sie im RCP8.5 am Ende des Jahrhunderts stark zunehmen. Eine ebensolche Tendenz ist auch bei den Spannbreiten der extremen Entwicklungspfade erkennbar, wobei das RCP8.5 diesbezüglich die größten Spannbreiten aufweist. Für das RCP2.6 kann die Schlussfolgerung gezogen werden, dass die klimatischen Randbedingungen zum Ende des 21. Jahrhunderts ungünstiger für die Grundwasserneubildung werden könnten. Die Mediane und ebenso die oberen und unteren Quartile werden von Periode zu Periode kleiner, d.h. sie weisen immer stärker in Richtung einer Abnahme der Grundwasserneubildung. Davon wären alle Regionen NRWs in ähnlichem Ausmaß betroffen. Der Ensembleteil RCP4.5 zeigt eher eine stabile Grundwasserneubildung auf einem leicht erhöhten Niveau. Im Ensembleteil RCP8.5 streuen die Änderungssignale der Grundwasserneubildung deutlich stärker, und auch extreme Entwicklungen können häufiger indiziert werden. Insgesamt ist keine eindeutig in allen Großräumen ausgeprägte einheitliche Richtung der Entwicklung zu erkennen. Tendenziell ist die Streuung im RCP8.5 stärker in den Großräumen ausgeprägt, in denen auch in den Ensembleteilen RCP2.6 und RCP4.5 größere Interquartilsabstände resultieren. Dies hat wahrscheinlich mehrere sich in ihrer Wirkung überlagernde Ursachen, die durch weitergehende Untersuchungen noch zu identifizieren wären. Insgesamt könnte die Schlussfolgerung gezogen werden, dass im Teilensemble RCP8.5 in allen zukünftigen Perioden eine höhere Grundwasserneubildung stattfinden könnte, jedoch die Unsicherheiten hinsichtlich der Ausprägung der klimatischen Randbedingungen am größten sind.

Die in den Großlandschaften regional unterschiedlichen Änderungen der Grundwasserneubildung (Abb. 4) haben zwei wesentliche Ursachen. Einerseits variieren die klimatischen Änderungssignale (Niederschlag und potenzielle Evapotranspiration) aus den RCMs auf der regionalen Skale. Änderungssignale des Niederschlags können beispielsweise in den Mittelgebirgen im Vergleich zu den Tieflandregionen unterschiedlich stark ausgeprägt sein. In den Karten der Abb. 2 sind solche Muster für viele Ensemblemitglieder erkennbar. Zusätzlich hat die regionale Häufigkeit spezifischer lokaler Standorteigenschaften (z. B. BFI-Werte im Festgesteinsbereich) einen Einfluss, wie stark sich die klimatischen Änderungssignale und ihre zeitlichen Muster auf die Grundwasserneubildung auswirken. Im Sandmünsterland dominieren beispielsweise künstlich entwässerte grundwasserbeeinflusste Standorte. Auf diesen Standorten findet zukünftig wahrscheinlich während der Sommermonate eine stärkere Grundwasserzehrung statt. Aufgrund eines projizierten höheren Niveaus der potenziellen Evapotranspiration im Sommerhalbjahr resultieren höhere mittlere Bodenfeuchtedefizite in der Wurzelzone über der Grundwasseroberfläche und damit höhere kapillare Aufstiegsraten, d.h. negative Grundwasserneubildung im Sommerhalbjahr. Höhere Winterniederschläge können diese Grundwasserzehrung nicht unbedingt vollständig ausgleichen (z. B. R85-MI5-CLM-mGROWA). In der Eifel fehlen solche Standorte weitgehend und es dominieren Festgesteinsstandorte. Eine sommerliche Grundwasserzehrung findet auf den Festgesteinsstandorten nicht statt und deswegen können höhere Winterniederschläge in der regionalen Bilanz netto zu einer geringeren Veränderung der Grundwasserneubildung führen. Die regional variierende nutzbare Feldkapazität der Böden hat ebenfalls einen Einfluss auf den Zeitpunkt des Beginns der Grundwasserneubildungsperiode und damit auf die regionale Ausprägung der Änderungssignale (Herrmann et al. 2017).

Als Resultat dieses vielfältigen Zusammenspiels tendieren letztendlich R85-MI5-CLM-mGROWA und R85CA2-CLM-mGROWA zu einem sehr starken Rückgang der Grundwasserneubildung, weil das projizierte Klima im Sommerhalbjahr zu einer stärkeren Grundwasserzehrung führt, der im Winterhalbjahr nur eine geringe Veränderung der Grundwasserneubildung gegenübersteht. Mit R85MP2-R09-mGROWA wird eine sehr starke Zunahme projiziert, weil vom RCM ein vergleichsweise starker Anstieg der Winterniederschläge projiziert wird, der viele andere Mechanismen überlagert. Die genannten Projektionen können als Begrenzungen der Bandbreite der projizierten Entwicklungspfade angesehen werden.

\section{Bewertung der Robustheit der Ensembleprojektion}

Die sich nun stellende Frage ist, wie die Robustheit der Ensembleprojektionen analysiert und bewertet werden kann. Diesbezüglich haben Pfeifer et al. (2015) eine statistische Methodik und Visualisierung mithilfe von Klimasignalkarten vorgeschlagen, sowie für Deutschland auf die Änderung saisonaler und extremer Niederschläge angewendet. Ebendiese Methodik wurde aus Gründen der Vergleichbar- 
keit mit denselben Entscheidungskriterien (Signifikanzniveaus) angewendet, um die Robustheit der projizierten Änderungssignale der Grundwasserneubildung zu bewerten. Pfeifer et al. (2015) definieren Robustheit als Kombination aus Übereinstimmung im Vorzeichen der projizierten Änderungssignale im gesamten Ensemble (test for agreement) und der statistischen Signifikanz der Änderungen in individuellen Projektionen (test for significance), d.h. die Bewertung erfolgt anhand von zwei zu erfüllenden Kriterien.

Der Test auf Übereinstimmung im Vorzeichen der Änderungssignale ist erfüllt, wenn jeweils mindestens $66 \%$ (vgl. Pfeifer et al. 2015) der Mitglieder in den RCP-Teilensembles eine $\mathrm{Zu}$ - oder Abnahme der 30-jährigen Mittelwerte der Grundwasserneubildung für die drei zukünftigen Perioden gegenüber der historischen Referenzperiode projizieren.

Der Test auf Signifikanz der Änderungssignale nutzt einen U-Test (Mann-Whitney-Wilcoxon-Test, Mann und Whitney 1947; Wilcoxon 1945). Dieser wurde durchgeführt, um zu überprüfen, ob die Verteilung der Jahressummen der Grundwasserneubildung in den drei zukünftigen Perioden signifikant von der Verteilung während der projizierten historischen Referenzperiode abweicht. Der Test auf Signifikanz ist erfüllt, wenn jeweils mindestens $66 \%$ der Mitglieder in den RCP-Teilensembles signifikante Änderungen in der Verteilung der Jahreswerte aufweisen. Die Wahrscheinlichkeit die Nullhypothese $\mathrm{H}_{0}$ (keine signifikante Änderung) fälschlicherweise zurückzuweisen wird auf $15 \%$ festgelegt (Signifikanzniveau $\alpha=0,15$ ).

Durch Auszählen der in Abb. 4 dargestellten Änderungssignale der Grundwasserneubildung lässt sich erkennen, dass trotz des relativ hohen Signifikanzniveaus von 0,15 keines der RCP-Teilensembles für keine der Perioden eine robuste $\mathrm{Zu}$ - oder Abnahme projiziert (Ergebnisse in Abb. 5).
In vielen Fällen wird das 66\%-Kriterium der Übereinstimmung nicht erreicht. Zusätzlich sind die Änderungen in der Verteilung nicht in einer ausreichend großen Zahl der Projektionen signifikant. Für die Jahressummen der Grundwasserneubildung in NRW werden erst ab mittleren $\mathrm{Zu}$ - oder Abnahmen von ca. $20 \mathrm{~mm} / \mathrm{a}$ statistisch signifikante Veränderungen indiziert. Dieser für NRW charakteristische Wert ist abhängig vom tatsächlichen Niveau der Grundwasserneubildung und kann demnach auf der regionalen Skale stärker variieren. Aus statistischer Perspektive kann für NRW die Schlussfolgerung gezogen werden, dass das Ensemble keine robuste Veränderung projiziert und deshalb langfristig eher eine Grundwasserneubildung auf dem historischen Niveau (am Ende des 21. Jahrhunderts) stattfinden wird.

Die bis hierher dargelegten Ergebnisse bedürfen abschließend einer überregionalen Einordnung. Jacob et al. (2014) zeigen europaweit die projizierte Änderung der Jahresniederschläge des EURO-CORDEX Ensembles für RCP4.5 und RCP8.5. Sie beschreiben eine Übergangszone, die auch westliche Teile der Bundesrepublik Deutschland umfasst, in der die projizierten Änderungssignale des Niederschlags von einer Abnahme (Süd- und Südwesteuropa) in eine Zunahme (Nord- und Nordosteuropa) wechseln. Im Bereich dieser Übergangszone werden keine robusten Änderungssignale des Jahresniederschlages projiziert, oder die Zu- bzw. Abnahmen sind weniger stark ausgeprägt. Pfeifer et al. (2015) haben die Robustheit der mit dem EURO-CORDEX Ensemble projizierten Änderung der Winterniederschläge deutschlandweit auf Ebene der Landkreise untersucht. Aus der Bilanzperspektive wirken sich höhere Winterniederschläge positiv auf die Grundwasserneubildung aus, diese Wirkung kann jedoch durch ein höheres Niveau der potenziellen Evapotranspiration im Herbst und Winter sowie durch höhere Bodenfeuchtedefi-

\begin{tabular}{|c|c|c|c|c|c|c|c|c|c|c|c|c|c|c|c|c|c|c|c|c|c|c|c|c|c|c|c|c|}
\hline \multirow{4}{*}{$\begin{array}{r}\text { Periode } \\
2011-2040\end{array}$} & \multirow{2}{*}{\begin{tabular}{|c|} 
RCP \\
RCP2.6
\end{tabular}} & \multicolumn{3}{|c|}{ BergL } & \multicolumn{3}{|c|}{ Eifel } & \multicolumn{3}{|c|}{ NiedB } & \multicolumn{3}{|c|}{ NiedT } & \multicolumn{3}{|c|}{ Sandm } & \multicolumn{3}{|c|}{ SaSie } & \multicolumn{3}{|c|}{ Weser } & \multicolumn{3}{|c|}{ WestB } & \multicolumn{3}{|c|}{ WestT } \\
\hline & & 8 & 22 & 1 & 9 & 11 & 11 & 1 & 0 & 22 & 1 & 0 & 3 & 8 & 22 & 22 & 8 & 2 & 11 & 8 & 2 & 22 & 8 & 2 & 3 & 78 & 2 & 22 \\
\hline & RCP4.5 & 0 & 20 & 30 & 80 & 0 & 30 & 90 & 0 & 4 & 0 & 0 & 50 & 0 & 20 & 0 & 0 & 0 & 30 & 90 & 0 & 0 & 0 & 0 & 30 & 30 & 0 & 30 \\
\hline & & 1 & 29 & 12 & 5 & 47 & 18 & & 18 & 18 & 82 & 18 & 8 & 76 & 24 & 12 & 1 & 9 & 18 & 82 & 18 & 12 & 88 & 12 & 18 & 71 & 9 & 1. \\
\hline \multirow{3}{*}{ 2041-2070 } & & 56 & 44 & 22 & 56 & 44 & 11 & 78 & 2 & 11 & 78 & 22 & 11 & 56 & 44 & 22 & 56 & 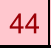 & 11 & 67 & 33 & 11 & 7 & 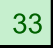 & 11 & 56 & 44 & 14 \\
\hline & CP4.5 & 50 & 50 & 30 & 60 & 40 & 10 & 8 & 20 & 30 & 70 & 30 & 40 & 60 & 40 & 20 & 50 & 50 & 10 & 70 & 30 & 20 & 60 & 40 & 30 & 60 & 40 & 40 \\
\hline & 5 & 71 & 29 & 2 & 1 & 2 & 35 & & 6 & 1 & 8 & 2 & 9 & 1 & 29 & 18 & P & 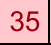 & 18 & 10 & 24 & 9 & 71 & 9 & 29 & 71 & 29 & \\
\hline \multirow{3}{*}{$2071-2100$} & .6 & 33 & 67 & 0 & 44 & 56 & 0 & 67 & 33 & 11 & 78 & 22 & 0 & 44 & 5 & 0 & 33 & 67 & 0 & 44 & 56 & 0 & 44 & 56 & 0 & 56 & 44 & 11 \\
\hline & CP4.5 & 70 & 00 & 20 & 60 & 40 & 10 & 80 & 20 & 10 & 90 & 10 & 20 & 60 & 40 & 10 & 60 & 40 & 20 & 60 & 40 & 40 & 60 & 40 & 20 & 50 & 50 & 10 \\
\hline & P8.5 & 47 & 5 & 7 & 59 & 41 & 65 & 8 & 18 & 47 & 65 & 35 & 47 & 53 & 47 & 47 & 65 & 35 & 47 & 65 & 35 & 53 & 59 & 41 & 47 & 53 & 47 & 53 \\
\hline
\end{tabular}

Abb. 5 Ergebnisse des Robustheitstests mit zwei Kriterien in den hydrogeologischen Räumen der Abb. 3. Ist das Kriterium erfüllt, sind die Zellen grün gefärbt, bei Nichterfüllung sind sie rot. Die ersten beiden Zellen enthalten jeweils die Prozentzahlen der Ensemblemitglieder mit Zu- bzw. Abnahme der Grundwasserneubildung. Die dritte Zelle enthält die Prozentzahl der signifikanten Änderungen

Fig. 5 Results of the 2-criteria robustness test (region names in Fig. 3). Green cells indicate positive and red cells negative test result. First two cells show percentage of ensemble members, which project rising and decreasing groundwater recharge. Third cell shows percentage of significant changes 
zite im Herbst teilweise oder auch vollständig aufgehoben werden (Herrmann et al. 2017). Für viele aber nicht alle Landkreise NRWs haben Pfeifer et al. (2015) eine robuste Zunahme der Winterniederschläge ermittelt. Dies spiegelt sich jedoch nicht in einer durch das Ensemble als robust projizierten Zunahme der Grundwasserneubildung wider. Vielmehr wird die Zunahme der Winterniederschläge eventuell knapp ausreichen, um andere eher negativ wirkende klimatische Einflüsse auf die Grundwasserneubildung und damit auf den Grundwasserhaushalt in regional unterschiedlichem Maße zu kompensieren. In anderen Regionen der Bundesrepublik Deutschland muss keine solche Kompensation resultieren, auch wenn robuste Zunahmen der Winterniederschläge projiziert werden. So werden wahrscheinlich $15 \%$ höhere Winterniederschläge (evtl. sind dies $20 \mathrm{~mm}$ ) im Mitteldeutschen Trockengebiet nicht ausreichen, um ein erhöhtes Niveau der potenziellen Evapotranspiration auszugleichen. Im Voralpenraum, bei deutlich höheren Winterniederschlägen und einer robusten Zunahme, könnte schlussendlich auch eine robuste Zunahme der Grundwasserneubildung projiziert werden. Exakt kann dies jedoch nur quantifiziert werden, wenn eine Modellkette inklusive eines spezialisierten Wasserhaushaltsmodells für die Projektionen verwendet wird. Im Süddeutschen Raum wird dazu beispielsweise das Modell GWN-BW (Gudera und Morhard 2015; Kopp et al. 2018) eingesetzt. Letztendlich lässt sich feststellen, NRW befindet sich wahrscheinlich in einer Übergangszone, in der eine robuste Zunahme der Winterniederschläge die Änderung von negativ auf die Grundwasserbilanz wirkenden Klimagrößen kompensieren kann, jedoch noch nicht als robuste Zunahme der Grundwasserneubildung wirksam wird.

\section{Ausblick}

Die Ergebnisse der Projektionen mit dem Multi-ModellEnsemble sowie der durchgeführte Robustheitstest liefern derzeit keine statistisch abgesicherte Begründung dafür, dass sich die langjährige mittlere Grundwasserneubildung in NRW systematisch und signifikant bis zum Ende des 21. Jahrhunderts ändern könnte. Nahezu alle Ensemblemitglieder projizieren auch mehrjährige Phasen mit „Grundwasserdürre“, vergleichbar mit der derzeitigen Situation in NRW, denen jedoch auch wieder feuchtere Dekaden mit einem überdurchschnittlichen Grundwasserneubildungsniveau folgen. Eine detaillierte Analyse der inner-dekadischen Variabilität der mit dem Ensemble projizierten Grundwasserneubildung steht noch aus und könnte wichtige Hinweise für notwendige Anpassungsmaßnahmen im Grundwassermanagement liefern.

Mit der Weiterentwicklung einzelner Komponenten der Modellkette werden zukünftig neue Projektionen möglich, die dann auch die Ergebnisse des hier präsentierten Robustheitstests ändern und eine Anpassung der damit verbundenen Aussagen notwendig machen können. Ebenso können andere Schlussfolgerungen aus dem Robustheitstest resultieren, wenn mehrere Projektionen zukünftig als nicht verlässlich identifiziert und eingestuft werden. Insofern empfiehlt sich eine kontinuierliche Aktualisierung und Erweiterung des Ensembles gemäß den Empfehlungen des Deutschen Wetterdienstes.

Die NRW-weiten Ergebnisse des Multi-Modell-Ensembles - die Grundwasserneubildung ist eines der Hauptergebnisse - sind als Datenbasis für weiterführende Studien mit Bezug zur Grundwasserbewirtschaftung verfügbar. Beispielsweise können mittlere und extreme Entwicklungspfade des Ensembles im Rahmen von Stresstests oder Betrachtungen zum zukünftigen Grundwasserdargebot Berücksichtigung finden. An dieser Stelle folgen wir den Empfehlungen aus dem Verbundprojekt ReKliEs-De (Huebener et al. 2017), das Ensemble und der Median der Änderungssignale zeigen die potenzielle Richtung zukünftiger Änderungen an. Es sollen jedoch in die Analysen auch immer extremere Entwicklungspfade einbezogen werden, weil für einzelne Entwicklungspfade derzeit keine verlässliche Eintrittswahrscheinlichkeit angegeben werden kann.

Danksagung Die Autoren danken dem Ministerium für Umwelt, Landwirtschaft, Natur- und Verbraucherschutz des Landes NordrheinWestfalen für die zur Verfügung gestellten Fördermittel.

Funding Open Access funding enabled and organized by Projekt DEAL.

Open Access Dieser Artikel wird unter der Creative Commons Namensnennung 4.0 International Lizenz veröffentlicht, welche die Nutzung, Vervielfältigung, Bearbeitung, Verbreitung und Wiedergabe in jeglichem Medium und Format erlaubt, sofern Sie den/die ursprünglichen Autor(en) und die Quelle ordnungsgemäß nennen, einen Link zur Creative Commons Lizenz beifügen und angeben, ob Änderungen vorgenommen wurden.

Die in diesem Artikel enthaltenen Bilder und sonstiges Drittmaterial unterliegen ebenfalls der genannten Creative Commons Lizenz, sofern sich aus der Abbildungslegende nichts anderes ergibt. Sofern das betreffende Material nicht unter der genannten Creative Commons Lizenz steht und die betreffende Handlung nicht nach gesetzlichen Vorschriften erlaubt ist, ist für die oben aufgeführten Weiterverwendungen des Materials die Einwilligung des jeweiligen Rechteinhabers einzuholen.

Weitere Details zur Lizenz entnehmen Sie bitte der Lizenzinformation auf http://creativecommons.org/licenses/by/4.0/deed.de.

\section{Literatur}

Ad-hoc-Arbeitsgruppe Hydrogeologie: Regionale Hydrogeologie von Deutschland - Die Grundwasserleiter: Verbreitung, Gesteine, Lagerungsverhältnisse, Schutz und Bedeutung. Geologisches Jahrbuch Reihe A, Bd. 163. Bundesanstalt für Geowissenschaften und Rohstoffe, Hannover (2016) 
ATV-DVWK, Deutsche Vereinigung für Wasserwirtschaft, Abwasser und Abfall: Verdunstung in Bezug zu Landnutzung, Bewuchs und Boden (2002)

Bloomfield, J.P., Allen, D.J., Griffiths, K.J.: Examining geological controls on baseflow index (BFI) using regression analysis: an illustration from the Thames Basin, UK. J Hydrol 373, 164-176 (2009)

Bogena, H., Kunkel, R., Schöbel, T., Schrey, H.P., Wendland, F.: Distributed modeling of groundwater recharge at the macroscale. Ecol Model 187, 15-26 (2005)

Disse, M.: Modellierung der Verdunstung und der Grundwasserneubildung in ebenen Einzugsgebieten. Fakultät für Bauingenieurund Vermessungswesen der Universität Fridericiana zu Karlsruhe, Karlsruhe (1995)

DKRZ: ESGF node at DKRZ (2018). https://esgf-data.dkrz.de/proje cts/esgf-dkrz/, Zugegriffen: 2018

DWD: Informationen zu den verfügbaren regionalen Klimaprojektionen (2019). https://www.dwd.de/DE/klimaumwelt/klimaforschung/ klimaprojektionen/fuer_deutschland/fuer_dtld_rcp-datensatz. html?nn=17770, Zugegriffen: 3. Dez. 2019

Ehret, U., Zehe, E., Wulfmeyer, V., Warrach-Sagi, K., Liebert, J.: Should we apply bias correction to global and regional climate model data? Hydrol Earth Syst Sci 16, 3391-3404 (2012)

Engel, N., Müller, U., Schäfer, W.: BOWAB - Ein Mehrschicht-Bodenwasserhaushaltsmodell. GeoBerichte 20, 85-98 (2012)

van Genuchten, M.T.: A closed-form equation for predicting the hydraulic conductivity of unsaturated soils. Soil Sci Soc Am J 44, 892-898 (1980)

Gudera, T., Morhard, A.: Hoch aufgelöste Modellierung des Bodenwasserhaushalts und der Grundwasserneubildung mit GWN-BW. Hydrol Wasserbewirtsch 59, 205-216 (2015)

Haberlandt, U., Klöcking, B., Krysanova, V., Becker, A.: Regionalisation of the base flow index from dynamically simulated flow components - a case study in the Elbe River Basin. J Hydrol Reg Stud 248, 35-53 (2001)

Hattermann, F., Huang, S., Koch, H.: Climate change impacts on hydrology and water resources. Meteorol Z 24, 201-211 (2015)

Herrmann, F., Chen, S., Heidt, L., Elbracht, J., Engel, N., Kunkel, R., Müller, U., Röhm, H., Vereecken, H., Wendland, F.: Zeitlich und räumlich hochaufgelöste flächendifferenzierte Simulation des Landschaftswasserhaushalts in Niedersachsen mit dem Model mGROWA. Hydrol Wasserbewirtsch 57, 206-224 (2013)

Herrmann, F., Hübsch, L., Elbracht, J., Engel, N., Keller, L., Kunkel, R., Müller, U., Röhm, H., Vereecken, H., Wendland, F.: Mögliche Auswirkungen von Klimaänderungen auf die Grundwasserneubildung in Niedersachsen. Hydrol Wasserbewirtsch 61, 245-261 (2017)

Herrmann, F., Keller, L., Kunkel, R., Vereecken, H., Wendland, F.: Determination of spatially differentiated water balance components including groundwater recharge on the federal state level-a case study using the mGROWA model in North Rhine-Westphalia (Germany). J Hydrol Reg Stud 4, 294-312 (2015)

Herrmann, F., Wendland, F., Wolters, T., Bergmann, S., Eisele, M.: Projektionen der Grundwasserneubildung unter dem Einfluss des Klimawandels in Nordrhein-Westfalen. Gewässerschutz Wasser Abwasser 251, 13 (2020)

Himmelsbach, T., Neukum, C.: Prognosen in der Hydrogeologie Kristallkugel oder fundierte Wissenschaft? Grundwasser 23(3), 207-208 (2018)

Holman, I.P., Allen, D.M., Cuthbert, M.O., Goderniaux, P.: Towards best practice for assessing the impacts of climate change on groundwater. Hydrogeol J 20, 1-4 (2012)

Huang, S., Hattermann, F.F., Krysanova, V., Bronstert, A.: Projections of climate change impacts on river flood conditions in Germany by combining three different RCMs with a regional eco-hydrological model. Clim Change 116, 631-663 (2013)
Huang, S., Krysanova, V., Österle, H., Hattermann, F.F.: Simulation of spatiotemporal dynamics of water fluxes in Germany under climate change. Hydrol Process 24, 3289-3306 (2010)

Hübener, H., Spekat, A., Bülow, K., Früh, B., Keuler, K., Menz, C., Radtke, K., Ramthun, H., Rathmann, T., Steger, C., Toussaint, F., Warrach-Sagi, K.: ReKliEs-De Nutzerhandbuch (2017)

Huebener, H., Hoffmann, P., Keuler, K., Pfeifer, S., Ramthun, H., Spekat, A., Steger, C., Warrach-Sagi, K.: Deriving user-informed climate information from climate model ensemble results. Adv Sci Res 14, 261-269 (2017)

Jacob, D., Petersen, J., Eggert, B., Alias, A., Christensen, O.B., Bouwer, L.M., Braun, A., Colette, A., Déqué, M., Georgievski, G., Georgopoulou, E., Gobiet, A., Menut, L., Nikulin, G., Haensler, A., Hempelmann, N., Jones, C., Keuler, K., Kovats, S., Kröner, N., Kotlarski, S., Kriegsmann, A., Martin, E., Meijgaard, E., Moseley, C., Pfeifer, S., Preuschmann, S., Radermacher, C., Radtke, K., Rechid, D., Rounsevell, M., Samuelsson, P., Somot, S., Soussana, J.-F., Teichmann, C., Valentini, R., Vautard, R., Weber, B., Yiou, P.: EURO-CORDEX: new high-resolution climate change projections for European impact research. Reg Environ Change 14, 563-578 (2014)

Knoche, R., Keuler, K.: Dynamische Regionalisierung. promet 99, 29-40 (2017)

Kopp, B., Baumeister, C., Gudera, T., Hergesell, M., Kampf, J., Morhard, A., Neumann, J.: Entwicklung von Bodenwasserhaushalt und Grundwasserneubildung in Baden-Württemberg, Bayern, Rheinland-Pfalz und Hessen von 1951 bis 2015. Hydrol Wasserbewirtsch 62, 62-76 (2018)

Kotlarski, S., Keuler, K., Christensen, O.B., Colette, A., Déqué, M., Gobiet, A., Goergen, K., Jacob, D., Lüthi, D., van Meijgaard, E., Nikulin, G., Schär, C., Teichmann, C., Vautard, R., Warrach-Sagi, K., Wulfmeyer, V.: Regional climate modeling on European scales: a joint standard evaluation of the EURO-CORDEX RCM ensemble. Geosci Model Dev 7, 1297-1333 (2014)

LANUV: Klimaatlas NRW (2020). https://www.klimaatlas.nrw.de/ Klimaprojektionen-Artikel, Zugegriffen: 12. Sept. 2020

Mann, H.B., Whitney, D.R.: On a test of whether one of two random variables is stochastically larger than the other. Ann Math Stat 18, 50-60 (1947)

Marke, T., Mauser, W., Pfeiffer, A., Zängl, G.: A pragmatic approach for the downscaling and bias correction of regional climate simulations: evaluation in hydrological modeling. Geosci Model Dev 4, 759-770 (2011)

Menzel, L., Thieken, A.H., Schwandt, D., Bürger, G.: Impact of climate change on the regional hydrology - scenario-based modelling studies in the German rhine catchment. Nat Hazards 38, 45-61 (2006)

Merz, B., Maurer, T., Kaiser, K.: Wie gut können wir vergangene und zukünftige Veränderungen des Wasserhaushalts quantifizieren? Hydrol Wasserbewirtsch 56, 244-256 (2012)

Milly, P.C., Betancourt, J., Falkenmark, M., Hirsch, R.M., Kundzewicz, Z.W., Lettenmaier, D.P., Stouffer, R.J.: Climate change. Stationarity is dead: whither water management? Science 319, 573-574 (2008)

Moriasi, D.N., Arnold, J.G., Liew, M.W.V., Bingner, R.L., Harmel, R.D., Veith, T.L.: Model evaluation guidelines for systematic quantification of accuracy in watershed simulations. Trans ASABE 50, 885-900 (2007)

Moss, R.H., Edmonds, J.A., Hibbard, K.A., Manning, M.R., Rose, S.K., van Vuuren, D.P., Carter, T.R., Emori, S., Kainuma, M., Kram, T., Meehl, G.A., Mitchell, J.F.B., Nakicenovic, N., Riahi, K., Smith, S.J., Stouffer, R.J., Thomson, A.M., Weyant, J.P., Wilbanks, T.J.: The next generation of scenarios for climate change research and assessment. Nature 463, 747-756 (2010)

Müller, U., Waldeck, A.: Auswertungsmethoden im Bodenschutz. Landesamt für Bergbau, Energie und Geologie Niedersachsen, Hannover (2011) 
Pfeifer, S., Bülow, K., Gobiet, A., Hänsler, A., Mudelsee, M., Otto, J., Rechid, D., Teichmann, C., Jacob, D.: Robustness of ensemble climate projections analyzed with climate signal maps: seasonal and extreme precipitation for Germany. Atmosphere 6, 677-698 (2015)

Rauthe, M., Steiner, H., Riediger, U., Mazurkiewicz, A., Gratzki, A.: A central European precipitation climatology part I: generation and validation of a high-resolution gridded daily data set (HYRAS). Meteorol Z 22, 235-256 (2013)

Renger, M., Bohne, K., Facklam, M., Harrach, T., Riek, W., Schäfer, W., Wessolek, G., Zacharias, S.: Bodenphysikalische Kennwerte und Berechnungsverfahren für die Praxis. In: Wessolek, G., Kaupenjohann, M., Renger, M. (Hrsg.) Bodenökologie und Bodengenese. Technische Universität Berlin - Fachgebiet Bodenkunde/ Standortkunde und Bodenschutz - Institut für Ökologie, Berlin (2009)

Scheihing, K.W.: Klimawandel in Niedersachsen und mögliche Folgen für die Grundwasserbewirtschaftung: ein Review. Hydrol Wasserbewirtsch 63, 85-97 (2019)

Schmidli, J., Frei, C., Vidale, P.L.: Downscaling from GCM precipitation: a benchmark for dynamical and statistical downscaling methods. Int J Climatol 26, 679-689 (2006)
Sørland, S.L., Schär, C., Lüthi, D., Kjellström, E.: Bias patterns and climate change signals in GCM-RCM model chains. Environ Res Lett 13, 74017 (2018)

Tetzlaff, B., Kuhr, P., Wendland, F.: Ein neues Verfahren zur differenzierten Ableitung von Dränflächenkarten für den mittleren Maßstabsbereich auf Basis von Luftbildern und Geodaten. Hydrol Wasserbewirtsch 52, 9-18 (2008)

Wilcoxon, F.: Individual comparisons by ranking methods. Biom Bull 1, 80-83 (1945)

Willkofer, F., Schmid, F.-J., Komischke, H., Korck, J., Braun, M., Ludwig, R.: The impact of bias correcting regional climate model results on hydrological indicators for Bavarian catchments. J Hydrol Reg Stud 19, 25-41 (2018)

Hinweis des Verlags Der Verlag bleibt in Hinblick auf geografische Zuordnungen und Gebietsbezeichnungen in veröffentlichten Karten und Institutsadressen neutral. 